\title{
Desenvolvimento de habilidades de lógica em estudantes do ensino médio: Uma proposta fundamentada na neurociência
}

Developing logic skills in high school students: A proposal based on neuroscience

\author{
N. S. Bastos ${ }^{1 *}$; D. F. Adamatti1 ; F. A. H. Carvalho² \\ ${ }^{1}$ Centro de Ciências Computacionais, Universidade Federal do Rio Grande, S/CEP,Rio Grande-RS,Brasil \\ ${ }^{2}$ Programa Pós-Graduação Educação em Ciências: Química da Vida e Saúde \\ Universidade Federal do Rio Grande, Rio Grande-RS, Brasil \\ *nanahbastos@yahoo.com.br
}

(Recebido em 22 de setembro de 2014; aceito em 29 de dezembro de 2014)

\begin{abstract}
Este artigo apresenta a proposta de treinamento da habilidade lógica em estudantes de ensino médio e sua análise baseada na neurociências. O foco da pesquisa está em verificar diferenças de gênero, ou seja, se as habilidades para as áreas de ciências exatas e engenharias podem ser ampliadas em meninas, e como essas atividades podem ser validadas a nível fisiológico, segundo a plasticidade cerebral.

Palavras-chave: Computação, Lógica, Neurociência
\end{abstract}

This paper presents a proposal for training of logical skill in high school students and its analysis based on neuroscience. The focus of the research is to ascertain gender differences, i.e., if the skills in the areas of engineering and sciences can be increased in girls, and how these activities can be validated at the physiological level, according to brain plasticity.

Keywords:Neuroscience, Logic, Computing

\section{INTRODUÇÃO}

O meio acadêmico revela diferenças de gênero no que tange a inserção masculina e feminina no campo das engenharias. Para [1] uma possível razão para essa diferença na participação é apontada por estudos que demonstram que as atitudes culturais também influenciam o desempenho das meninas em ciências e "mesmo meninas que apresentam ótimo desempenho em ciências no ensino médio têm menos confiança em sua capacidade de serem bem-sucedidas nas disciplinas universitárias de ciências e, por tanto, são menos propensas a se especializar em ciências no ensino superior." As autoras, retomando os estudos de [5] destacam que variações no modo como os meninos e meninas resolvem problemas podem ser responsáveis pelas diferenças de desempenho entre os sexos no ensino médio. Os meninos, por exemplo, podem ser melhores na identificação de estratégias efetivas para resolver os tipos de problemas que costumam aparecer em testes padronizados de matemática. Salientam também os achados de [13], os quais apontam que o fato de meninos passarem mais tempo jogando videogame do que as meninas pode explicar parte dessa diferença, pois "a prática de videogames melhora o desempenho das mulheres em testes de percepção espacial, fator importante no desempenho em matemática". Esse resultado aponta que a prática de jogos eletrônicos pode ajudar as meninas a desenvolver as habilidades cognitivas espaciais.

Sem dúvida, o cérebro é modificável pela cultura, pois diante da plasticidade neuronal é possível reconfigurar as redes neurais e reconstruir conhecimento. $O$ autor [12] destaca que "a inteligência humana é altamente maleável. Pode ser moldada e incrementada por meio de vários tipos de intervenções".

A fase da adolescência é um momento de intenso desenvolvimento cerebral. Estudos recentes empregando imagens cerebrais mostraram que existe uma maturação contínua no córtex pré-frontal durante a idade adulta jovem, principalmente em resultado da maior mielinização, o que indica que os axônios se desenvolvem, participando de um número maior de sinapses (comunicações entre os neurônios e, portanto, ampliando conexões 
neurais). Esse aumento de circuitos frontais na adolescência pode sustentar o desenvolvimento de novas capacidades cognitivas [8][10].

Conforme [7] os adolescentes processam informações com mais rapidez, utilizam recursos de processamento com mais eficiência, tem maior flexibilidade cognitiva. Tal eficiência do controle cognitivo na adolescência coincide com mudanças biológicas no desenvolvimento cerebral. Os autores lembram que Piaget atribuía as mudanças no pensamento adolescente ao surgimento das operações formais.

Segundo [1], o estágio operatório-formal, propicia aos adolescentes pensar logicamente sobre idéias que não estão relacionadas a referentes concretos no mundo real. "Tipicamente esse estágio é definido como o período durante o qual os adolescentes desenvolvem esquemas operatórios que lhes permitem raciocinar logicamente sobre conceitos abstratos." Ainda conforme as autoras, todos os adolescentes e adultos neurologicamente saudáveis têm capacidade para lógica formal, mas somente aqueles cujas situações de vida exigem essa capacidade vão realmente desenvolvê-la e a experiência é um componente essencial do desenvolvimento cognitivo.

De acordo com [8]: "o pensamento atual argumenta que a maneira pela qual o pensamento do adolescente se desenvolve depende muito do conteúdo dos problemas que os adolescentes encontram e dos contextos em que se encontram." Daí a importância de oferecer atividades de intervenção. Uma alternativa é usar ferramentas computacionais como suporte ao ensino.

Desse modo, espaços educativos podem utilizar a tecnologia como ferramenta a fim de oportunizar o desenvolvimento das capacidades cognitivas das meninas. Nesse panorama emergem as seguintes questões: Como proporcionar o desenvolvimento de competências lógicas nas meninas? As ferramentas tecnológicas podem contribuir para esse desenvolvimento?

Diante dessas considerações, o artigo apresentado mostra-se relevante, uma vez que ao investigar o potencial do treinamento de lógica computacional, contribui para qualificar recursos humanos no campo das ciências exatas e engenharias.

Este artigo está estruturado da seguinte forma: na seção 2 são apresentados dados de estudos que mostram a baixa inserção das mulheres no meio acadêmico das engenharias, além disso, aborda possíveis razões para essa questão. Em seguida, na seção 3 são abordadas características cerebrais e a capacidade lógica. A seção 4 detalha a metodologia usada para o estudo e os avanços da pesquisa. Na seção 5 são discutidos os resultados preliminares, em que são analisados um teste de lógica e um questionário de perfil aplicados em um grupo piloto.

\section{A INSERÇÃO DAS MULHERES NO MEIO ACADÊMICO DAS ENGENHARIAS}

Em geral, sabe-se que as mulheres começam a habilidade da fala e letramento mais cedo que os homens, porém quando atinge a idade adulta estas habilidades se igualam. $\mathrm{O}$ que no entanto, ocorre de maneira diferente para as áreas de matemática e ciências em que as meninas começam com boa habilidade nos primeiros anos de escola e declinam a medida que progridem em direção à sua profissão. Ainda assim, alguns estudos mostram que as mulheres superam os homens em cálculos, bem como na habilidade de somar ou dividir uma série de números rapidamente, entretanto, eles acabam se saindo melhor na maioria dos testes matemáticos, incluindo geometria, mensuração, probabilidade e charadas matemáticas. Estes dados são observados por [4] onde considera os dados do National Assesment of Educational Progress (NAEP), que testa inúmeros alunos dos Estados Unidos. As meninas tiram notas mais baixas em matemática e ciências na $4^{\mathrm{a}}$ e $8^{\mathrm{a}}$ séries, ficando ainda mais para trás na $12^{\mathrm{a}}$ série, fato que era ainda maior há uma ou duas décadas.

Em seus estudos, [4] salienta que as mulheres compõem somente $20 \%$ dos cursos de engenharia, e em ciência da computação a proporção de mulheres diminuiu de um pico de $37 \%$ em meados da década de 1980 para $28 \%$ em 2002. Para [1], uma possível razão para 
essa diferença é apontada por estudos que demonstram que as atitudes culturais também influenciam o desempenho das meninas em ciências e "mesmo meninas que apresentam ótimo desempenho em ciências no ensino médio, têm menos confiança em sua capacidade de serem bem-sucedidas nas disciplinas universitárias de ciências e, por tanto, são menos propensas a se especializar em ciências no ensino superior".

Segundo [1], destacam que variações no modo como os meninos e meninas resolvem problemas podem ser responsáveis pelas diferenças de desempenho entre os sexos no ensino médio. Os meninos, por exemplo, podem ser melhores na identificação de estratégias efetivas para resolver os tipos de problemas que costumam aparecer em testes padronizados de matemática, salientam ainda o fato de os meninos passarem mais tempo jogando videogame do que as meninas, fato que pode explicar parte dessa diferença, pois "a prática de videogames melhora o desempenho das mulheres em testes de percepção espacial, fator importante no desempenho em matemática" [1]. Esse resultado aponta que a prática de jogos eletrônicos pode ajudar as meninas a desenvolver as habilidades cognitivas espaciais. A habilidade espacial requer treinamento, sendo assim a brecha entre os sexos pode ser reduzida, brincadeiras com blocos, bolas, jogos de computadores, videogames e muitas outras brincadeiras podem contribuir para essa diferença. Ainda que, os meninos tenham algumas vantagens no que diz respeito a habilidade espacial, esta não é a única barreira encontrada para que as meninas possam atingir seu potencial em matemática e ciências, também existem barreiras culturais. Sem dúvida, o cérebro é modificável pela cultura, pois diante da plasticidade neuronal é possível reconfigurar as redes neurais e reconstruir conhecimento.

\section{A ATIVIDADE CEREBRAL E A CAPACIDADE LÓGICA}

O cérebro é a parte mais importante do sistema nervoso, pois é através dele que obtemos consciência das informações que chegam pelos órgãos dos sentidos e processamos essas informações, comparando-as com nossas vivências e expectativas, sendo capaz de modificar nossos comportamentos e aprender, à ainda os processos mentais como o pensamento, capacidade de julgamento e a atenção são resultados do seu funcionamento [3].

Estas capacidades só são possíveis porque são feitas através de circuitos nervosos, os neurônios. Um neurônio é capaz de disparar impulsos seguidamente, muitas vezes por segundo, mas as informações só podem ser transmitidas para outra célula, por meio do axônio, que é uma estrutura que ocorre geralmente nas porções finais do prolongamento neural. Sendo o local por onde a passagem da informação entre as células, chamado de sinapses, e a comunicação é feita através da liberação de um neurotransmissor.

Segundo [3] no que diz respeito a nossa espécie sabe-se que não existem dois cérebros iguais, mas pode-se afirmar que "todos temos vias motoras e sensoriais que seguem o mesmo padrão." E que estas vias "estão previstas nas informações genéticas de nossas células e são construídas enquanto nosso organismo se desenvolve dentro do útero materno".

O sistema nervoso inicia sua formação nas primeiras semanas de vida embrionária, tendo a forma de um pequeno tubo em que a parede é formada por células-tronco que darão origem aos neurônios e também à grande maioria das células auxiliares, denominadas de células gliais, encontradas no adulto. Os autores, destacam ainda que em uma primeira fase, o evento mais importante é a continua divisão das células-tronco, formando assim novos neurônios que, em um curto prazo de tempo irão aumentar significativamente seu numero inicial reduzido, chegando a bilhões.

À medida que a região que formará o cérebro vai ser formando, os novos neurônios irão inicialmente deslocar-se para os lugares em que estão geneticamente predeterminados para ocupar. Logo após cumprida a fase de diferenciação, tem-se início a formação das conexões com os demais neurônios, para que assim sejam criados os circuitos necessários para executar diferentes funções. Ao fim, devidamente posicionados, emitem prolongamentos e tem seus axônios nos lugares estabelecidos, ocorre então a sinaptogênese, que é a formação das sinapses que irão completar efetivamente os circuitos nervosos. No entanto, é fundamental o cuidado nessa fase inicial do desenvolvimento do embrião, pois erros 
ocorridos nessa fase, causados por problemas genéticos ou ambientais, poderão ter como conseqüência distúrbios ou incapacidades para a vida toda. Ainda, é preciso salientar a afirmação feita por [3] que diz que "A interação com o ambiente é importante porque é ela que confirmará ou induzirá a formação de conexões nervosas e, portanto, a aprendizagem ou o aparecimento de novos comportamentos que delas decorrem." [3], pois o sistema nervoso nos primeiros anos de vida apresenta o maior índice de plasticidade, a capacidade de formação de novas sinapses é muito grande, o que é justificado pelo longo período de maturação do cérebro, que se estende até os anos de adolescência. Porém, ainda que com um índice relativamente baixo em adultos, a plasticidade nervosa ocorre por toda a vida, concluindo assim que a capacidade de aprendizagem é mantida, ainda que exija um pouco mais de tempo e esforço para que ocorra.

De todo processo de desenvolvimento do sistema nervoso, um dos períodos mais importantes é o que corresponde a época da adolescência, quando um grande rearranjo tem lugar, havendo um acelerado processo de eliminação de sinapses, um "desbastamento sináptico", que ocorre em diferentes regiões do córtex cerebral [3]. As modificações que ocorrem na adolescência preparam o indivíduo para a vida adulta. $\mathrm{O}$ aumento das células corticais é progressivo durante o período da infância, mas declina na adolescência até atingir o padrão adulto, o que reflete na otimização do potencial de aprendizagem. Nesta fase diminui a capacidade de aprendizagem de novas informações, mas há um ganho na capacidade de elaborar e utilizar o que já foi aprendido.

\section{MATERIAIS E MÉTODOS}

Este artigo tem como foco o ensino de lógica para alunos do ensino médio e a coleta e análise dos sinais cerebrais antes e após o treinamento, como forma a verificar mudanças cerebrais fisiológicas nos estudantes. Assim, espera-se verificar a plasticidade do cérebro, bem como comprovar que não há diferenças fisiológicas entre gêneros para ensino e motivação para as áreas de ciências exatas e engenharias.

Para atingirmos esse objetivo, os seguintes passos serão realizados:

1. Escolha do grupo de alunos a serem analisados (dois anos finais do ensino médio). Testes de coeficiente de inteligência devem ser aplicados, pois alunos fora da média (superdotados ou subdotados) podem influenciar nos resultados obtidos. Serão selecionados 3 meninos e 3 meninas;

2. Aplicação de testes de lógica no grupo de alunos a serem analisados e coleta de sinais cerebrais durante sua execução, de forma a verificar quais regiões do cérebro são "ativadas" durante essa atividade;

3. Oficina de ensino de lógica computacional, utilizando a linguagem Scratch, com duração de 24 horas/aulas, ministradas em 2 meses;

4. Aplicação de novos testes de lógica no grupo de alunos e coleta de sinais cerebrais;

5. Análise completa dos dados.

Até o momento, já foram realizadas as atividades 1 e 2 . A atividade 3 está em andamento. No entanto as análises dos sinais cerebrais ainda não foram feitas, pois o software está em estudo.

\subsection{FERRAMENTA SCRATCH}

O Scratch é uma ferramenta criada pelo Grupo do MIT (Massachusetts Institute of Technology), em colaboração com o grupo de Yasmin Kafai na UCLA (University of California, Los Angeles). É um ambiente e linguagem de programação destinada a programadores iniciantes, usado para o aprendizado de conceitos matemáticos $\mathrm{e}$ computacionais. $\mathrm{O}$ Scratch substitui o código digitado por uma abordagem de "arrastar-esoltar" que facilita sua manipulação. A ferramenta enfatiza a manipulação de mídias e apóia as atividades de programação que despertam interesse em jovens, tais como a criação de 
histórias animadas, jogos e apresentações interativas. Porém para o desenvolvimento de projetos na ferramenta exige que se tenha uma compreensão sobre lógica.

\section{RESULTADOS E DISCUSSÃO}

Através da análise com o grupo de 23 alunos, composto por 13 meninas e 10 meninos, estudantes do primeiro ano ensino médio, foi aplicado um teste de lógica e um questionário de perfil a cada aluno, antes de iniciar o treinamento com a ferramenta Scratch. Com isso, pode-se observar que a maioria dos alunos do sexo masculino tem uma boa freqüência ao acesso a jogos, principalmente jogos de tiro e corrida, ambos exercitam de maneira boa a percepção espacial, já as meninas jogam pouco, e em geral jogam "social game" ou jogos sociais, que demandam de baixo envolvimento emocional dos jogadores e é de fácil aprendizado. Os alunos, em maioria, alegam não gostar da matéria de matemática, ainda que gostem de fazer desafios de lógica.

Notou-se também que, apenas uma menina quer cursar curso superior na área das exatas, e que cinco meninos querem exatas, apenas um humanas e os restante não sabe ainda o que quer. Apesar de significativas diferenças entre os resultados do questionário, em que as meninas alegam não ter interesse pela área das exatas, estes se contrapõem com os resultados dos testes de lógica, em que as meninas tiveram melhor resultado em relação aos meninos. Este fato possivelmente pode ser justificado pela falta de confiança das meninas na área das exatas, o que, de acordo com [4], pertence a forte barreira cultural, em que o estereótipo de que as mulheres não tem a mesma capacidade na área que os homens, é fortemente disseminado, comprovando assim, que a baixa confiança das meninas em matemática é um fator significativo para seu desempenho. A figura 1 mostra a primeira página do teste de lógica aplicado aos alunos, sendo possível visualizar apenas duas questões. O teste é formado por um total de quatro questões. 


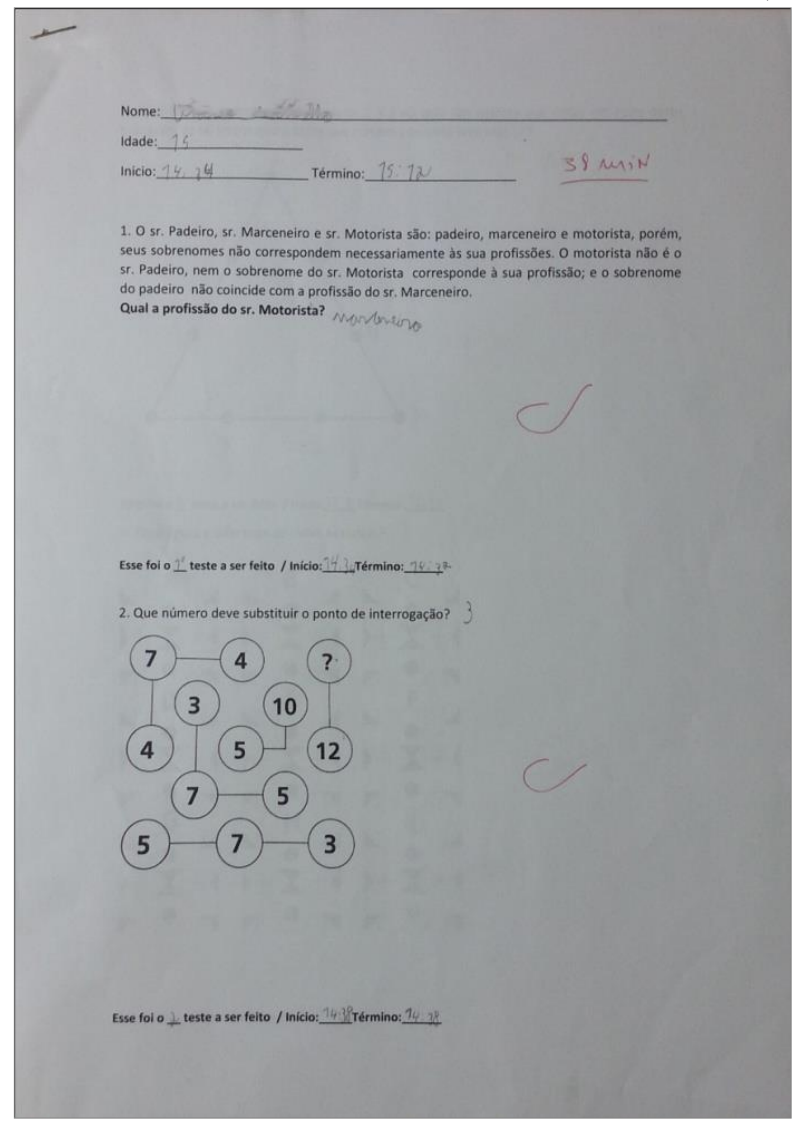

Figura 1: Teste de lógica 1 (pré treinamento)

Para uma melhor compreensão dos resultados observados, a figura 2 apresenta um gráfico comparativo dos resultados dos testes entre meninas e meninos, em relação ao numero de acertos e a média do tempo para a execução do teste, juntamente com o numero de alunos que querem cursar exatas.

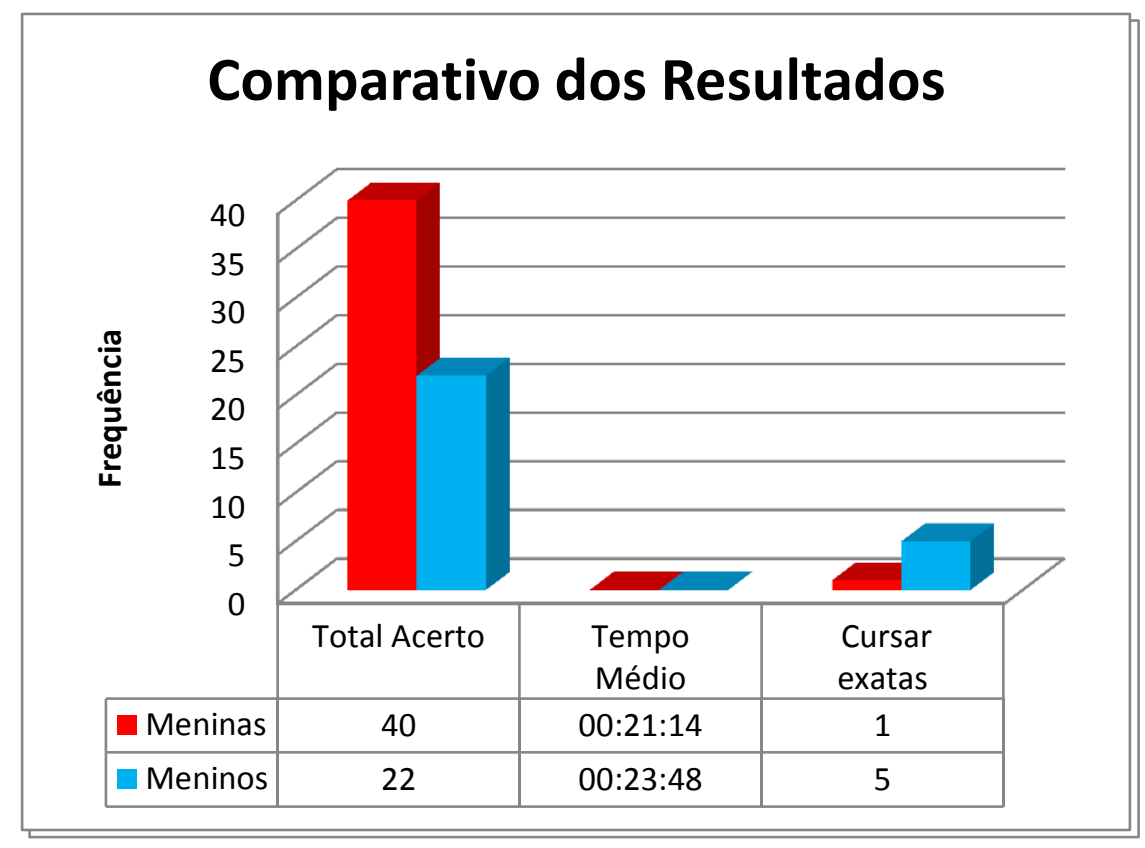

Figura 2: Quadro comparativo entre sexo 


\section{CONCLUSÃO}

Ainda que os índices de inserção de mulheres no nível superior nas áreas de exatas, como engenharia é baixo, as meninas iniciam os estudos com bons resultados em matemática, havendo um forte declínio a medida que se aproximam do momento de transição para o meio acadêmico, existindo assim um numero significativo maior de homens. Estudos observam que essas diferenças se justificam pelo fato de os homens apresentarem um melhor desempenho com habilidades matemáticas que as mulheres, estas diferenças podem ser fortemente influenciadas por questões culturais.

Neste trabalho, pode-se observar uma significativa diferença nos resultados dos testes e questionário, entre sexo. Em que, apesar de as meninas não praticarem jogos e não terem interesse pela área das exatas, se saíram melhor que os meninos em relação ao numero de acerto e tempo. O que, pode ser justificado por uma possível falta de confiança.

Pretende-se ainda, para as próximas análises, coletar os sinais cerebrais durante a execução dos testes de lógica antes do grupo iniciar o curso e ao final, podendo então de fato observar as reais áreas do cérebro que sofrem alguma modificação com o treinamento de exercícios usando lógica computacional.

\section{REFERÊNCIAS BIBLIOGRÁFICAS}

1. Boyd D, Bee H. Lifespan Development, $6^{\text {th }}$ Edition, 2011.

2. Carvalho FAH de. Reaprender a Aprender: a pesquisa como alternativa metacognitiva. Tese (Doutorado em Educação). 2007. Faculdade de Educação, Pontifícia Universidade Católica do Rio Grande do Sul, Porto Alegre. 150p.

3. Cosenza RM, Guerra LB. Neurociência na Educação, Porto Alegre, ed. Artmed, 2011.

4. Eliot L. Cérebro azul ou rosa: $\mathrm{O}$ impacto das diferenças de gênero na educação. Tradução: Marina Adriana Veríssimo Veronese; revisão técnica: Jane Felipe. 2013. Porto Alegre: Penso.

5. Gallagher S. How The Body Shapes The Mind, 2005.

6. Galiazzi M do C. Seria tempo de repensar as atividades experimentais no ensino de Ciências. Educação, 2000.

7. Gazzaniga MS, Ivry RB, Mangun GR. Neurociência Cognitiva. 2008. Porto Alegre: Artmed.

8. Gazzaniga MS, Heatherton T. Psychological Science: Mind, Brain, and Behavior. WW Norton, New York, 2007. 3rd Edition.

9. Gil AC. Didática do Ensino Superior. 2006. São Paulo: Atlas.

10. Herculano-Houzel S. Fique de Bem com seu Cérebro. 2007. Ed. Sextante.

11. Kolb B, Whishaw IQ. Neurociência do Comportamento. 2002. São Paulo: Manole.

12. Sternberg RJ. Psicologia Cognitiva. Trad. AM Luche, R Galman, 2005. Cengage Learning, São Paulo.

13. Terlecki MS, Newcombe NS. How important is the digital divide? The relation of computer and videogame usage to gender differences in mental rotation ability. Sex Roles. 2005; 53(5-6):433-441. 